\title{
Una Aproximación al Factor Humano desde el Enfoque de Recursos y Capacidad
}

\author{
José Sänchez Pérez \\ Universidad de Cádiz
}

RESUMEN

Este trabajo estudia las posibilidades de "recursos humanos" de fornarse un acriu'n estratégico en" el marco teórico de la visión de la empresa basada en recursas y' capacialades. El propósito de este articulo es doble. Primero valorar si recursos bumanos tiene consideracion estratégica dentro de este enfoque; es decir, si supera ciertas condiciones de escasez. valia, beterugeneidad, dificultad para la imitación, etc. Y'segundo, facilitar una nueva via para la valoracion del impacto organizativo de la gestión de los recursos bumanos. Para ello, en primer lugar se exponen los principios generales del marco conceptual elegido. mas tarde se analiza el encaje del capital humano en dicbo contexto teórico y finalmente se elabora un modelo que explicita las posibilidades y conexiones del capital bumano cara a la ventaja competitiva sostenible.

Palabras clave: Factor Humano, Capacidad, Recursos Estratégicos.

ABSTRACT

This article analizes bow buman resources to became a strategic asset under the principles of the Resource-based View of the Firm. This paper embodies two puposes. First, to determine whetber buman resources bave actually strategic consideration under the Resource-based Vieu. because certain conditions must be met to generate the sustainable competitive advantage. And second. to facilitate a new way to evaluate the organizational human resource management impact. In order to achieve this, first. the foundaments drau from the Resource-based Vieu are shoun. Second. the paper analizes the human resources as considered as scarce, valuable, firm-specific. and difficult to imitate ssets. Finally, a model is created in order to develop a better understanding of linkages between buman resources and competitive advantage.

Key words: Resources Human. Capability. Strategic Resources. 


\section{INTRODUCCIÓN}

Este trabajo pretende analizar, y en su caso ubicar, dentro de la visión de la empresa basada en los recursos y capacidades dinámicas uno de las figuras más reconocidas y controvertidas de nuestros días: el factor humano. Así, en primer lugar, se expone sucintamente los fundamentos de esta integradora aproximación, para más tarde analizar este objeto material bajo el enfoque de recursos y capacidades dinámicas. Finalmente, se elabora un modelo en el que situar y comprender las conexiones del factor humano con la competitividad y la satisfacción del accionista.

\section{RECURSOS Y CAPACIDADES CON RELACIÓN A LA COMPETTTINIDAD.}

La palabra mágica de nuestro <<status quo>> socio-económico es competitividad; rasgo propio de los agentes exitosos en sus relaciones de concurrencia, aplicable a los individuos en el marco organizativo y, sobre todo, a las empresas en relación con su industria. De esta última acepción, se deriva el establecimiento de condiciones idóneas. para la supervivencia de una compañía, siempre en rivalidad con otras (Salas, 1996).

De acuerdo con la teoría basada en los recursos y capacidades, las organizaciones procuran desarrollar ventajas competitivas sostenibles a partir de características únicas, las cuales no provienen del entorno, sino del interior de la firma; de su singular dotación de activos. Esta especificidad resultante del proceso de acopio de recursos es la que permite el desarrollo diferenciado de estrategias generadoras de rentas sostenibles en el tiempo: ya sea liderazgo en costes (ventaja competitiva basada en la oferta) o diferenciación (ventaja competitiva basada en la demanda).

$\mathrm{Y}$, si la ventaja competitiva reside en la habilidad para anticiparse y satisfacer las necesidades de los clientes mediante productos elaborados con recursos y capacidades <<únicos〉>, el desafío académico consiste en detectar y analizar tales fuentes de unicidad (Ulrich y Lake, 1990). Para determinar las capacidades distintivas resultan de utilidad los procesos organizativos (valoración relativa de las capacidades), la dotación de recursos existente (potencial de capacidades) y la trayectoria recorrida por la firma (capacidades latentes) (Teece, Pisano y Schuen, 1997).

Así, una primera aproximación adelanta que la presencia de recursos distribuidos heterogéneamente con movilidad imperfecta es capaz de generar competencias distintivas sobre las que fundamentar ventajas competitivas sostenibles (Wernerfelt, 1984; Barney, 1989, 1991; Grant, 1991; Peteraf, 1993; Dierickx y Cool, 1989). Esta imperfección del mercado constituye la auténtica piedra angular de esta teoría.

Entre las imperfecciones del mercado, también denominadas barreras <<ex ante $>>$ a la competencia (Peteraf, 1993), suele citarse la complejidad social, la ambigüedad causal y las condiciones históricas únicas (Barney, 1991). Conviene remarcar que constituyen éstas el auténtico germen de la heterogeneidad en la dotación de recursos de las organizaciones; si bien tampoco hay que dramatizai 
excesivamente, pues no son absolutamente impermeables o infranqueables; todas tienen una determinada altura y cierta ratio de erosión (Reed y DeFillippi, 1990).

Cuando el análisis se efectúa sobre las competencias, las limitaciones anteriores se tornan imposibilidades. Ello es debido a la inexistencia de un mercado al cual acudir para adquirir competencias. Y, considerando que son éstas el soporte de la ventaja competitiva, es posible sentenciar negativamente sobre la existencia de un mercado de factores estratégicos (Barney, 1989). El razonamiento que suele acompañar a esta negación es de una contundencia similar: puede resultar viable adquirir todos los recursos, de manera individualizada, que son usados por las competencias; pero no se puede captar la competencia, la singular experiencia de la empresa en el manejo de tales recursos. En consecuencia, la única alternativa posible para disponer de factores estratégicos reside en la acumulación interna (Diericks y Cool, 1989). Y este proceso, aparte de requerir tiempo, posee otras características relevantes: deseconomías en la comprensión del tiempo necesario para su desarrollo, economías derivadas del nivel de recursos disponibles y ambigüedad causal.

Pero también es preciso señalar que en entornos cambiantes como el actual. la agilidad organizativa materializada en la capacidad de absorber el cambio determinará las posibilidades de éxito y supervivencia (Spoor, 2000). Es necesaria la renovación de dichas capacidades para mantener la sintonía con el ambiente. Ello implica la necesidad de salvar las rigideces, técnicas y cognitivas, que frenan esta renovación de competencias (Leonard-Barton, 1992) porque éstas han de ser necesariamente creadas en el interior de la firma: no existe un mercado de capacidades. En este proceso de renovación de sus propias capacidades las firmas transforman recursos y capacidades existentes que se desarrollaron en su día y más tarde dejaron de ser usadas (Garud y Nayyar. 1994). En consecuencia, la historia de una firma puede ser origen de barreras (inercias) al cambio y, al mismo tiempo, generadora de recursos (facilitadora) para la construcción de nuevas capacidades dinámicas.

\section{Aportaciones relevantes: Barney, Grant y Peteraf}

Llegados a este punto, queda por precisar cuál o cuáles son los atributos que, marcan diferencias con relación a la capacidad de favorecer la captación de rentas en el tiempo. Los autores que han llevado a cabo esta tarea han seguido una metodología similar, si bien los resultados alcanzados muestran leves diferencias.

Barney (1986a, 1991) entiende que las exigencias a superar son cuatro: valioso, o útil para la concepción o ejecución de estrategias; escaso, o no disponible fácilmente, no perfectamente imitable y carente de sustitutos estratégicamente equivalentes.

Grant $(1991,1995)$ desarrolla un ejercicio idéntico con resultados muy coincidentes. A su entender los recursos generan competitividad si y sólo si son 
escasos y valiosos; y mantienen la ventaja competitiva si superan además exigencias de durabilidad, transparencia, dificultad de transmisión y réplica y apropiación.

La aportación de este autor no es baladí pues arroja mayor clarividencia al proceso investigador iniciado: desliga los factores que posibilitan la competitividad de aquellos otros que la hacen perdurable en el tiempo, lo cual reviste gran interés.

De este modo, para que un recurso contribuya al sostenimiento de la competitividad ha de ser perdurable en el tiempo, pues difícilmente recursos efímeros puedan sostener ventajas competitivas duraderas; mínimamente opaco, lo que dificulta la percepción del génesis de la competitividad; difícilmente transferible, lo que dificulta a los competidores apropiarse de activos causales (inmovilidad geográfica, información imperfecta, especificidad e inmovilidad de capacidades); difícilmente imitables, que resulte espinoso tornarlo en objeto de réplica y facilitadores de la captación de rentas.

Peteraf (1993) estima que son cuatro las bases fundamentales que propician y sostienen la ventaja competitiva de una firma:

1) Heterogeneidad de los recursos. Las compañías con recursos singulares tratan de sustentar en éstos su ventaja competitiva, una vez comprobado que se adecúan a las oportunidades reinantes en el ambiente (Thompson y Strickland, 1990).

2) Limitación a la competencia ex-post. Se trata de beneficiarse de ciertos mecanismos de aislamiento que impidan a empresas competidoras acceder a un nivel de rentas similar por imitación o aplicación de otros recursos valiosos (Rumelt, 1974). Esta condición abarca tanto el concepto de barrera de entrada de Porter, como el de barrera a la movilidad de Wernerfelt.

3) Limitación a la competencia ex-ante. Esta condición resalta la necesidad de que el mercado de factores sea imperfecto; es decir, que no todas las empresas puedan acceder en las mismas condiciones a estos peculiares recursos.

4) Movilidad imperfecta. En efecto, para que la empresa pueda apropiarse de la totalidad de, o al menos comparta, las rentas generadas por los factores que posee o controla; éstos han de presentar resistencias a ser objeto de transacciones comerciales, sea por motivos reglamentarios o de especificidad.

Por supuesto, es preciso que estas cuatro condiciones convivan simultáneamente de modo que la heterogeneidad de los recursos genere rentas, ya sean ricardianas $\bullet$ monopolísticas; los límites ex-post levanten barreras, con las que asegurar el nivel de rentas alcanzado de los ataques de los competidores; los límites ex-ante orienten la viabilidad financiera de las estrategias potenciales, evitando incurrir en costes. superiores al potencial de generación de rentas; $y$, por último, la movilidad imper fecta salvaguarde en el tiempo, al menos en parte, las rentas alcanzadas. 


\section{El factor humaNo COMO RECURSO ESTRATÉGICO}

Las razones sostenidas por Jalbert (Thierry, 1994) para la integración de los recursos humanos en el proceso estratégico -internacionalización de los mercados, aceleración de las innovaciones, escasez y encarecimiento de los recursos financieros, acentuación de la división internacional del trabajo, turbulencia e incapacidad de previsión del entorno, reducción de los costes de producción, exigencias crecientes de calidad y de servicio al cliente, gestión más ajustada de efectivos, ascenso masivo de los niveles de cualificaciones y adaptación de las estructuras de funcionamiento de las empresasson, valga el juicio de valor, contundentes y variadas; pero lo realmente interesante es que el simple hecho de plantear este estadío evolutivo supone reconocer que los recursos humanos de una firma tienen posibilidades de convertirse en factor distintivo ya sea en el diseño o en la implantación de la estrategia.

En efecto, el capital humano, considerado como el "conjunto de aptitudes, conocimientos y saber hacer de los individuos" (Vateville, 1985) es ya, en mayoría de empresas, el recurso fundamental. Incluye los contactos y relaciones personales, además de otras cualidades individuales como reputación, lealtad, polivalencia o flexibilidad (Fernández, Montes y Vázquez, 1999). En definitiva, el factor humano se presta perfectamente a ser gestionado como un activo generador de ventajas competitivas sostenibles (Swiercz y Spencer, 1992; Pfeffer, 1994; Huselid, 1995).

Del mismo modo que Barney (Mueler, 1996) demostró que la cultura organizativa puede tornarse fuente de ventaja competitiva sostenible -los valores y creencias son realmente difíciles de cambiar-, caso de optar por el factor humano para tan elevado propósito, resulta vital conocer cuáles son sus posibilidades. Este análisis será desarrollado a continuación siguiendo el enfoque antes presentado, basado en la noción de exigencias aplicadas a los recursos:

a) Heterogeneidad, escasez y valía. Estos tres requisitos han sido agrupados con base en las relaciones que entre sí mantienen; pues un recurso es valioso si añade valor, pero no basta con ello; es preciso ser escaso, para evitar situaciones de posesión generalizada que no conducen a nada. Luego, la escasez se apoya en la existencia de trabas a la movilidad, que también son explicativas de la heterogeneidad.

La valía es plenamente reconocida en la literatura, pues disponer de personal cualificado cuya capacidad cognitiva le permita adquirir conocimientos y habilidades añadirá valor, vía eficiencia, innovación o creatividad; y todo ello sin desdeñar la incidencia de comportamientos excelentes derivados de actitudes de alineación.

Un recorrido por las singularidades de este activo permite constatar la superación de los criterios de valía, escasez y heterogeneidad (Sánchez, 1993): antes tómese conciencia de que la relación que les liga a la firma no está basada en la propiedad (Fournies, 1990); y que el sometimiento al proceso productivo es voluntario, a pesar de la existencia de contratos de trabajo. Continúese resaltando que su naturaleza es 
intangible, explicitada sólo_a través del comportamiento, pues, aunque los individuos sean seres tangibles, sus aportaciones organizativas, conocimientos, destrezas o habilidades, experiencias y actitudes, como la lealtad o la disposición al cambio, son elementos de indiscutible esencia intangible (Bonache, 1996; Grant, 1991).

Por otra parte, el reparto de estos intangibles entre la población no es equitativo; más bien se acomoda a una distribución normal, con las implicaciones que esto conlleva a la hora de tratar la presencia de estos activos en las compañías (Boxall, 1998). Finalícese arguyendo que su valor aumenta con su utilización; mediante la repetición y la experimentación, son susceptibles de ser mejorados, ya sea perfeccionando sus competencias o detectando sus potenciales. O sea, que no se vuelven obsoletos; o al menos, no tienen por qué (Wright, McMahan y McWilliams, 1994).

Son escasos, a pesar del entorno de paro generalizado predominante, porque no todos poseen las mismas competencias ni tampoco las organizaciones demandan perfiles homogéneos. Esta paradoja (Gabarro, 2000) del mercado laboral potencia la heterogeneidad en la dotación de este activo entre empresas competidoras. No es de extrañar pues que la noción de capital humano responda mejor a la idea de recurso generado internamente, como efecto directo de actividades propias, que a la de activo presente en un mercado eficiente de factores (Fernández, Montes y Vázquez, 1999).

Además, en este análisis habría que añadir el rasgo de la unicidad: cada personá es irrepetible, tanto en el espacio como en el tiempo. Este aserto, sin embargo, no ha incitado al abandono del análisis de la conducta humana; sino que aceptándole como premisa fundamental, se han obtenido valiosas conclusiones. Así, por ejemplo, existe una serie de rasgos comunes y aparentemente contradictorios en la conducta humana, tales como egoísmo y consideración hacia los demás; pereza y creatividad; etc. Cohen (1991) señala que las investigaciones realizadas en este sentidø desembocan en tres procesos principales: causalidad -existencia de algún agente desencadenante-, orientación -conducta encaminada hacia un fin- y motivación impulso, deseo o necesidad al que se le considera la base del comportamiento-.

Son seres complejos omnipresentes a escala organizativa: aparecen en todo el espectro de tecnologías, productos y mercados (Wright, McMahan y McWilliams, 1994). También podría decirse sobre ellos que son los únicos dotados de iniciativa y: creatividad, facultades éstas que los erigen en el activo imprescindible de cualquier proceso organizativo. Por todo ello, se acepta como prioridad fundamental de la estrategia de recursos humanos la conservación de los perfiles que se revelan necesarios para la viabilidad de la firma; si bien, es preciso reconocer que no todos los empleados de una compañía, sobre todo en el caso de una gran firma, constituyen materializaciones de activos escasos y valiosos. Se distingue así el personal <<nije clear>> o crítico para la creación de valor, del personal apoyo (Boxall, 1998).

Boxall (1998), centrándose en el personal nuclear, realiza otra distinción entre el personal interno y el externo. El primero se caracteriza por su habilidad para tomat 
decisiones estratégicas básicas como las que dan lugar a innovar exitosamente o imitar con excelencia. El rasgo principal que define al segundo reside en su capacidad para satisfacer a los clientes y su compromiso de estabilidad para con la firma. En resumen, mientras el primer grupo suministra a la firma capacidad de adaptación, el segundo suministra credibilidad para la capacidad operativa. Por tanto, ambos colectivos se tornan críticos, y a la vez complementarios, para el éxito de la compañía.

b) Movilidad imperfecta. Llegados a este punto hay que diferenciar dos aspectos: las barreras a la movilidad de los efectivos y las referidas al capital humano; porque no siempre "fichar" a la persona equivale a disfrutar de las ventajas que ésta generaba en su emplazamiento anterior. Es decir, para que el recurso humano sea objeto de libre intercambio entre empresas, es exigible la ausencia de barreras de movilidad. tanto para los efectivos, en su condición de tangible portador; como para el capital humano, auténtico activo estratégico.

En este sentido, la fidelidad se torna un aspecto crucial, y depende de las decisiones que tome el individuo, de acuerdo con su percepción de satisfacción interna y de oportunidades externas. El primer pensamiento al tratar la cuestión sobre las barreras de movilidad de los individuos sostiene que los activos humanos difícilmente contribuirán a ventajas sostenibles; pues, en principio, su libertad de movilidad es total (Mueler, 1996). Y en un mercado libre como es el laboral. caracterizado genéricamente por el intercambio humano voluntario, es de imaginar que las empresas tienten a los mejores empleados de sus competidores (Coff, 1997); ya que, como la teoría de la agencia pregona, nunca puede ser presumido un solapamiento suficiente entre los intereses de empleados y empleadores. De todas formas, cuando exista este solapamiento o mutualidad, ya aparece la primera barrera a la movilidad: la coincidencia de intereses; de igual forma, el conocimiento personal de los empleados de los competidores es un factor de erosión importante de este tipo de barreras.

Pero estas tentaciones no surten el efecto que en un mercado de libre competencia podría esperarse simplemente porque no lo es; más bien es un mercado con intensas fricciones internas y externas. En este sentido, las barreras que dificultan la movilidad de los efectivos entre compañías están levantadas sobre la admiración que sienten por los recursos no humanos -aspectos institucionales como cultura y legislación y organizativos como los mecanismos de fidelización (stock options, pensiones, primas de antigüedad, etc.)- (Fernández, Montes y Vázquez, 1999); y sobre la inercia que padecen los individuos para afrontar el cambio, aunque sólo sean cambios domésticos (Boxall, 1998). Los motivos son variados: responsabilidades familiares, identificación geográfica-cultural, inserción social, obligaciones laborales, etc.

También la propia cultura corporativa complementar las acciones anteriores haciendo más eficiente el sistema de contratación o emitiendo señales precursoras a candidatos potenciales que sean percibidas y valoradas por éstos como un elemento 
reductor del grado de asimetría de información referido al comportamiento futuro: de la firma en materia de relàciones laborales (Fernández, Montes y Vázquez, 1999).

c) Imitación imperfecta. La facultad para replicar una ventaja competitiva pasa por ser capaz de identificar con toda precisión los activos que generan tal capacidad. y, además, ser capaz de reproducir con exactitud sus componentes y las circunstancias bajo las que éstos funcionan, amén de gozar de contactos personales que suministren feed-back a este proceso (Fernández. Montes y Vázquez, 1999).

Este comentario genérico aplicado al capital humano y, de acuerdo con Grant (1991), requiere para su aplicación superar férreos mecanismos de aislamiento: con:diciones históricas únicas, complejidad social y ambigüedad causal. Para una justa valoración de la efectividad de esta barrera, téngase en cuenta que el capital humano no sólo es difícil de transmitir inter-organizaciones; sino que es incluso compleja. su reproducción interna con intenciones de extensión a otras divisiones o departa: mentos. Además, el potencial imitador se enfrenta, cuando trata de replicar capitạl humano, con serias adversidades derivadas de los fenómenos conocidos como "de:seconomías de comprensión" y "eficiencia de masa" (Dierickx y Cool, 1989)

Por supuesto, los procesos de réplica de otros activos no ofrecen estas resistencias. Así, por ejemplo, el capital tecnológico, de no ser por las patentes, resultaría relativamente fácil de copiar por los competidores mediante el espionaje industriall; la ingeniería inversa o el fichaje de cerebros (Fernández, Montes y Vázquez, 1999).

d) Sustitución imperfecta. Cuando un activo se convierte en sostén principal de una ventaja competitiva, la amenaza de un sustituto estratégicamente equivalente puede tornarlo obsoleto para este menester.

En este sentido, los recursos humanos cuentan con una doble ventaja. En primer lugar, poseen una habilidad inherente, fuera del alcance de cualquier otro activo, cual es la cognitiva. Este rasgo, por otra parte, les hace transferibles y adaptables a una gran variedad de tecnologías, productos y mercados. En segundo lugar, las prácticas de: recursos humanos permiten salvaguardar y potenciar el capital humano de la firma, evitando así la obsolescencia de los mismos (Carmona, Céspedes y Jerez, 1999).

e) Apropiación. Se trata de constatar la ausencia de barreras para cuando lí organización intente apropiarse del valor añadido que los recursos humanos han aportado al negocio. Estas barreras curiosamente estarían promovidas por la voluin: tad de los propios recursos humanos que, a veces sabedores de su valía estratégica demandan a la firma una mejor posición; lo cual, obviamente, afecta al grado di apropiación de rentas que la firma alcanza (Boxall, 1998).

Los contratos laborales, en contra de lo que espontánea y subconscientement pudiera asumirse, no constituyen para nada garantía de apropiación; pues sus dá̧ü sulas son negociables y, en cualquier caso, las partes tienen derecho de rescisióng 
contribuyen, en cambio, la escasa transparencia de las relaciones de propiedad sobre las rentas generadas a partir del concurso de activos intangibles, así como la participación global o mayoritaria de los efectivos en la generación de estas rentas (Carmona, Céspedes y Jerez, 1999).

\section{COMPETITIVIDAD Y RECURSOS HUMANOS.}

Este auténtico"eslabón perdido" ha sido objeto de numerosos estudios por parte de investigadores y estudiosos del tema. Sirva de punto de partida la reflexión que se deriva de la siguiente ilustración.

\section{Gráfico 1.}

Recursos Humanos y Ventaja Competitiva Sostenible

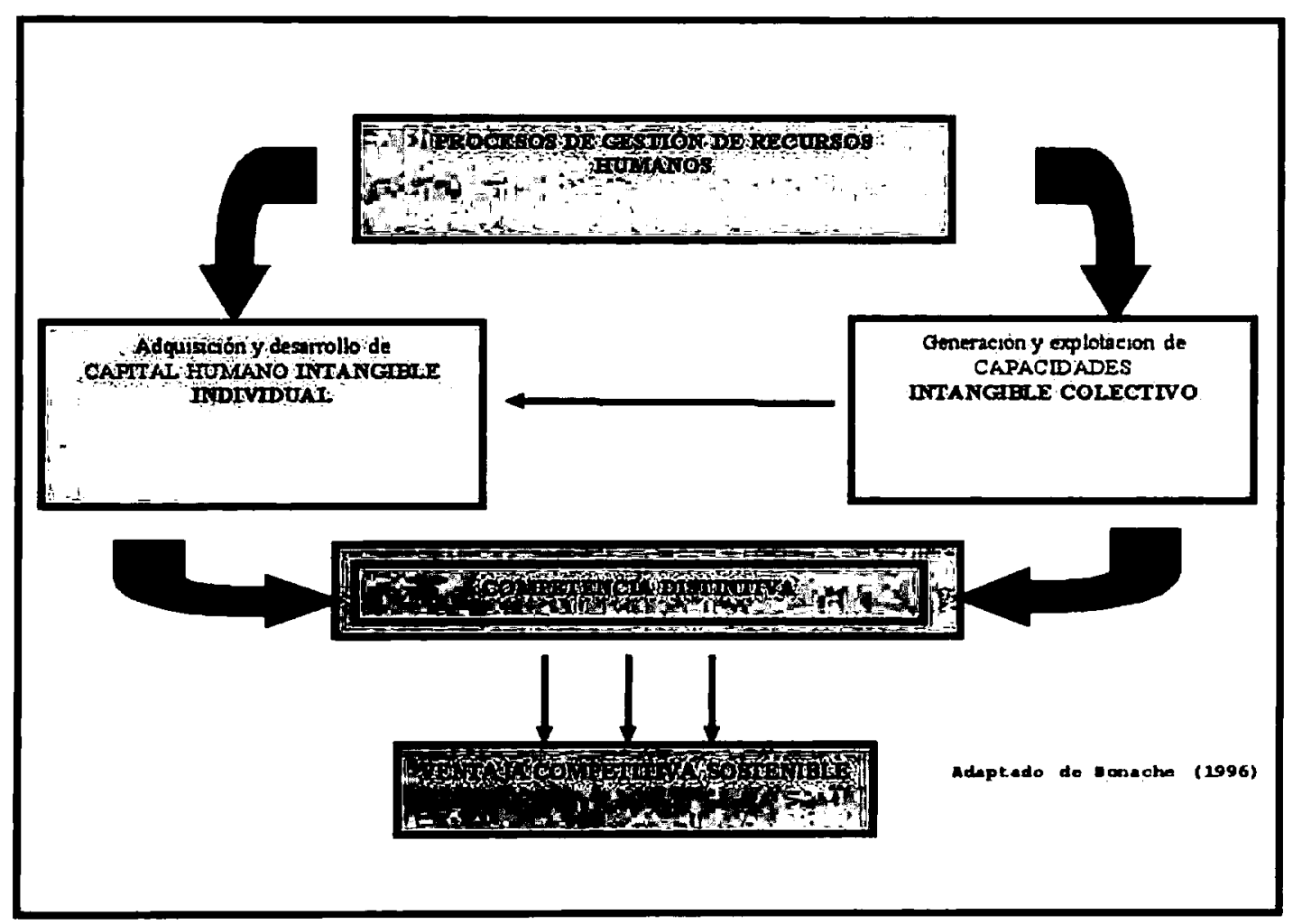

E incrementando un poco más el grado de abstracción y complejidad, introduciendo al mismo tiempo aportaciones de autores afanosos también por conocer las conexiones existentes entre competitividad y recursos humanos (Coff, 1997; Becker y Gerhart, 1996; Dyer y Reeves, 1995; Hammer y Barbera, 1997; Lado y Wilson, 1994; Miller, 1991; Schneider y Bowen, 1993; Schuler y Jackson, 1987; Williams, 1996; Wright, McMahan y. McWilliams, 1994; Yeung y Berman, 1997) aparece la siguiente figura: 


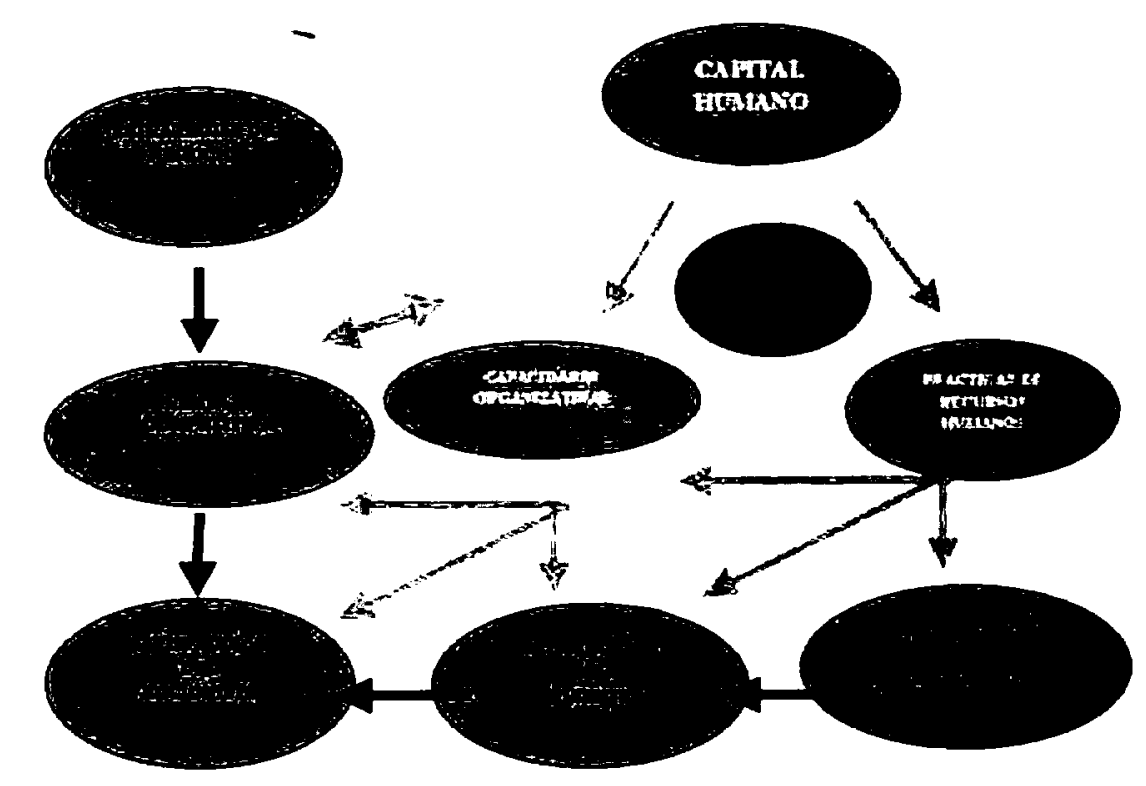

Fuente: Elaboración propia

Destáquese en este modelo el protagonismo de recursos humanos desde la ma: terialización de la idea de "Capital Intelectual" (Edvinsson y Malone, 1997) desglosa: do en su triple vertiente: capital humano, capital estructural y capital relacional. En este sentido es digna de destacar la contribución del capital relacional para con la satisfacción del empleado o la configuración del capital estructural bajo la noción de capacidades organizativas acumuladas en el tiempo.

La conexión prácticas de recursos humanos con capacidades organizativas se justifica a partir del ajuste que intencionadamente pretenderán los procesos de ges: tión de personal -rediseño, externalización, reducción, eliminación o automatiża: ción- para contribuir al sostenimiento y desarrollo de las competencias distintivas êt las que la organización fundamenta su ventaja competitiva. En este caso la alternati va que procede resaltar es aquélla que hace girar la estructura y la cultura organiza tiva alrededor de los equipos de trabajo y del trabajo en equipo respectivamente:.

La conexión prácticas de recursos humanos con satisfacción de los clientes se tomá inevitable en un entorno exigente de calidad. Admite dos vías de actuación: una porla que los clientes alcanzan sus objetivos, y otra por la que se crean y fortalecen relacio: nes personales, allende del carácter estrictamente profesional. En este sentido pueder ser destacados los equipos de ventas o comerciales y de atención al cliente.

Schneider y Bowen (1993) señalan que existe una relación directa y significature entre las actitudes de los empleados con relación a su trabajo y la valoración de servicio por parte del cliente. Por tanto, como las conductas de las personas no soi manipulables directamente, la solución que resta consiste en gestionarlas indirenta mente vía prácticas de recursos humanos, la cual habrá de orientarse a los aspeet 
distintivos que generen la ventaja competitiva deseada y duradera: calidad, cordialidad, rapidez, comodidad de acceso y estancia, etc.

Además, estas capacidades generadas, sostenidas y depuradas por recursos humanos se caracterizan por su aparición compleja e incremental; son competencias sensibles a la calidad de procesos tan diversos como reclutamiento, formación y construcción de equipos, programas de compensación y congruencia entre filosofía de la dirección y prácticas realizadas (Boxall, 1998).

$\mathrm{Si}$, asimismo, a partir de su intencionada persistencia, estas prácticas establecen y consolidan gradualmente lo que se denomina "arquitectura social", que se entiende como cierto grado de colaboración espontánea a partir de actividades de formación formales e informales continuadas, conocimiento tácito acumulado y fluidez de las relaciones internas; se genera un mix-social que sí puede llegar a constituir un elemento diferencial. Una materialización concreta de esta generalidad la constituyen las políticas que culminan consolidando actitudes tendentes a la mejora continua. Son pues estas singularidades las que hacen escasas y difíciles de imitar las prácticas que las generan. Y disfrutan de mecanismos de aislamiento porque se manifiestan a partir de diferentes procesos, disminuyendo la probabilidad de interpretación única y certera.

La existencia del capital humano, del intangible individual, es requisito imprescindible; pero no suficiente. Se requiere, además, realizar una dirección efectiva de este activo estratégico. Esta dirección será la responsable tanto de la acumulación y coordinación del capital humano para generar el intangible colectivo, amén de orientar su comportamiento hacia los objetivos de la firma.

En definitiva, son las prácticas de recursos humanos las que atraen, desarrollan y retienen el capital humano fuente de las capacidades distintivas; y este talento generador, por día más escaso en el actual y limitado mercado laboral, ha de ser atraído, motivado y conservado mediante prácticas innovadoras, preferiblemente también distintivas, como el reclutamiento electrónico, estructuras basadas en equipos, modelos alternativos de distribución del tiempo de trabajo, etc. (Hammer y Barbera, 1997). Y todo ello con la finalidad de alcanzar posteriormente la coordinación con otros activos que les haga valiosos, difíciles de transmitir, de imitar y de sustituir.

\section{Consideraciones finales}

Tras el análisis realizado, se pueden extraer las siguientes conclusiones:

a) El factor humano supera holgada y brillantemente las exigencias que la visión de la empresa basada en recursos y capacidades plantea a los activos destinados a convertirse en la base de la estrategia elegida. En consecuencia, puede conformar, por sí sólo o en combinación con otros activos, un aspecto singular explicativo de la existencia de diferenciales inter-organizaciones en el proceso de apropiación de rentas financieras provenientes del mercado. 
b) Si antes Salas (1996) apostaba por los recursos intangibles, desde el prisma de la competitividad mantenida, tras el análisis efectuado, se progresa en la precisión: es la participación del intangible colectivo en combinaciones de activos el que cuen: ta con más posibilidades de producir capacidades distintivas. Con ello se quiere enfatizar la contribución de los recursos que actúan conjuntamente, incluyéndose el factor humano en este marco y flexibilizando el abanico que propuso Mueler (1996)

c) El aprovechamiento del intangible colectivo que representa toda plantilla nọ está exento de dificultades; exige superar ciertos obstáculos: diseño ambiental que propicie su desarrollo, cierto compromiso de la dirección y considerables esfuerzos de construcción que combinen las disponibilidades de recursos con el aprendizaje $y$ asunción de nuevos roles. Y aquí irrumpe, con planteamientos disyuntivos y selecti. vos, el papel de la dirección.

\section{BiBLIOGRAFÍA}

AMIT, R. y SCHOEMAKER, P.J.H. (1993): “Strategic assets and organizational rent” Strategic Management Joumal. Vol. 14. Pág. 33-46.

ANDREWS, K.R. (1977): El concepto de la estrategia de la empresa. Pamplona: Ediciones Universidad de Navarra.

ANSOFF, H.I. (1965): Corporate Strategy. Harmondsworth, Reino Unido: Penguin.

BADEN-FULLER, C. y VOLBERDA, H. (1997): "Dormant capabilities, complex organi? sations and renewal" Ponencia presentada en la 17th Strategic Management Society Conference. Barcelona.

BARNEY, J.B. (1986a): "Types of Competition and the Theory of Strategy: Toward an Integrative Framework" Academy of Management Review. Vol. 11, №4. Pág. 791-800 BARNEY, J.B. (1986b): "Strategic factor markets: Expectations, luck, and business strate gy" Management Science. Vol. 32, № 10. Pág. 1321-1341.

BARNEY, J.B. (1986c): "Organizational culture: can it be a source of competitive advan: tage? Academy of Management Review No 11. Pág. 656-665.

BARNEY, Jay (1989): "Asset Stocks and Sustained Competitive Advantage: A Comment". Management Science. Vol. 35. № 12. Pág. 1511-1513.

BARNEY, J. (1991): “Firm Resources and Sustained Competitive Advantage , Journal of Management. Vol. 17, №1. Pág. 99-120;

BARNEY, J.B. y OUCHI, W.G. (Eds.) (1986): Organizational Economics. San Francised Jossey-Bass Publishers.

BECKER, B. y GERHART, B. (1996): "The Impact of Human Resource Management on Organizational Performance: Progr4ess and Prospects" Academy of Managementios urnal. No 39. Pág. 779-801.

BONACHE, J.(1996): "El papel de las políticas de recursos humanos en la internacios 
nalización de la empresa. Una aplicación al caso español. Economia Industrial. $\mathrm{N}^{\circ}$ 307. Pág. 37-48.

BOXALL, Peter (1998): "Achieving Competitive Advantage through Human Resource Strategy: Towards a Theory of Industry Dynamics" Human Resource Management Review. Vol. 8, № 3. Pág. 265-288.

CARMONA MORENO, Eva; CÉSPEDES LORENTE, JOsé J. y JEREZ GÓMEZ, Pilar (1999): "El valor de los recursos humanos en el desarrollo de capacidades estratégicas" II Workshop Internacional: La organización del futuro en la sociedad de la información: Gestión del cambio, recursos humanos y estructura. Del 25 al 28 de mayo. Cádiz.

CARRASCOSA, José Luis (1991): INFORMACCION: De la Era Industrial a la Sociedad de la Información. Madrid: Espasa-Calpe. Pág. 174.

CONNER, K.R. (1991): “A Historical Comparison of Resource-Based Theory and Five Schools of Thought Within Industrial Organization Economics: Do We Have a New Theory of the Firm?", Journal of Management. Vol. 17, No1. Pág. 121-154;

CORELLA, Angel (1994): "Cultura de Recursos Humanos" Capital Humano, № 56, Febrero. Pág. 19-23.

CORNELLA, Alfons (1994): Los recursos de la Información. Madrid: McGRAW-HILL.

DIERICKX, I. y COOL, K. (1989): "Asset stock accumulation and sustainability of competitive advantagen Management Science. Vol. 35. No 12. Pág. 1504-1511.

EDVINSON, Leif y MALONE, Michael S. (1997): El capital intelectual. Barcelona: Gestión 2000.

FERNÁNDEZ RODRÍGUEZ, Z. y SUÁREZ GONZÁLEZ, I. (1996): "La estrategia de la empresa desde una perspectiva basada en los recursos", Revista Europea Dirección y Economía de la Empresa. Vol. 5, № 3. Pág. 73-92

FERNÁNDEZ SÁNCHEZ, E.; MONTES PEÓN, J.M. y VÁZQUEZ ORDAS, C.J. (1999): "LOS recursos intangibles como factores de competitividad de la empresa" Dirección, Organización y Administración de Empresas. Revista electrónica. [Pág. Web]. bttp:// wuw.cepade.es/revistal

FERNÁNDEZ, Z. (1993): "La organización interna como ventaja competitiva para la empresa" Papeles de Economia Española. № 56. Pág. 178-193.

FOURNIES, Ferdinand F. (1990): Técnicas de dirección de personal. Cómo instruir para aumentar el rendimiento. Barcelona: McGraw-Hill. Pág. 52-54.

GABARRO, S. (2000): La paradoja del desempleo" Cinco Días. 9 de mayo. Pág. 21.

GARCIA ECHEVARRIA, Santiago (1994): "Exigencias de la dirección de los recursos humanos a los sistemas de información en la empresa" Alta Dirección. № 175 (Ed. especial, I Parte). Mayo-Junio.

GARUD, R. y NAYYAR, P. (1994): "Transformative Capacity: Continual Structuring by Intertemporal Technology Transfer" Strategic Management Journal. Vol. 15. Pág. 365-385.

GAUTIER, M. y LUPE, M.C. (1975): Les tableaux de bord de la fonction personnel: Entreprise Moderne d'Edition. Citado por VATEVILLE, E. (1985). 
GRANT, R.M. (1991): «The Resource-Based Theory of Competitive Advantage: Implications for Strategy Formulation", California Management Review. Vol. 33, № 3. Primavera. Pág. 114-135.

GRANT, R.M. (1995): Contemporary Strategy Analysis, segunda edición, Blackwell Publishers, Cambridge, Massachusets. Existe traducción al castellano: Dirección Estratégica, Editorial Civitas, Madrid, 1996.

GROE, Gerald M; PYLE, Willeam; JAMROG, Jay J. (1996):

"Information Technology and HR" Human Resource Planning. Vol. 19. N 1. Pág. 60-68.

HALL, R. (1993): "A framework linking intangible resources and capabilities to sustainable competitive advantage" Strategic Management Journal. Vol. 14. Pág. 607-618.

HAMEL, G. y PRAHALAD, C. K. (1990): "El propósito estratégico" Harvard Deusto Business Review. $1^{\circ}$ trimestre. Pág. 75-94.

HAMEL, G. y PRAHALAD, C. K. (1995): Compitiendo por el futuro. Barcelona: Ariel.

HAMMER, Leslie B. y BARBERA, Karen M. (1997): "Toward an integration of alternative work schedules and human resource systems" Human Resource Planning. Vol. 20 No 2. Pág. 28-36.

HITT, M.A.; KEATS, B.X. y DEMARIE, S.M. (1997): "Navigating in the new competitive landscape: Building strategic flexibility and competitive advantage in the 21st century" Working Paper, Workshop on "The impact of strategy, job design and organization structure on HRM: Scenarios for the year 2000". Departamento de Organización de Empresas. Universidad de Cádiz, 25-28 de mayo de 1997.

HOGLER, R.L.; HENLE, C. y BEMUS, C. (1998): "Internet Recruiting and Employment Discrimination: A Legal Perspective" Human Resource Management Review. Vol. 8, N². Pág. 149-164.

JACOBSON, Robert (1992): "The "Austrian" School of Strategy" Academy of Management Review. Vol. 7. No 4. Pág. 782-807;

LADO, Augustine A. Y WILSON, Mary C. (1994): "Human Resource Systems and Sustained Competitive Advantage: A Competency-based Perspective" Academy of Management Review. Vol. 19. No 4. Pág. 699-727.

LATTMANN, Charles y GARCIA ECHEVARRIA, Santiago (1992): Management de los recursos bumanos en la empresa. Cómo dirigir hombres en una nueva cultura corporativa. Madrid: Diaz de Santos.

LEONARD-BARTON, D. (1992): "Core Capabilities and Core Rigidities: A Paradox in Managing New Product Development" Strategic Management Journal. Vol. 13, Número especial de verano. Pág. 111-125.

LEONTIEF, W. W. (1968): "Domestic Production and Economics Trade", en R. CAVES y H. JOHNSON. (Eds.): Readings in International Economics. Illinois: Irwin.

LOPEZ SINTAS, Jordi (1996): "Los recursos intangibles en la competitividad de las empresas. Un análisis desde la teoría de los recursos" Economía industrial. № 307. Pág. 25-29 
MAHONEY, J.T. y PANDIAN, J.R. (1992): "The resource-based view within the conversation of strategic management", Strategic Managemenı Journal. Vol. 13. Pág. 363-380;

MARTINSONS, Maris G. (1994): "Benchmarking human resource information systems in Canada and Hong Kong" Information E Management. Vol 26, №6. Junio. Pág. 305-317. MILGROM, P. y ROBERTS, J. (1993): Economia. organización y gestión de la empresa. Barcelona: Ariel.

MONTGOMERY, C.A. (Ed.) (1995): Resouce-based and Evolutionary Theories of the Firm. Towards a Synthesis. Kluwer Academic Publishers, Norwell, Massachusetts;

MUELER, F. (1996): "Human resources as strategic assets: an evolutionary resourcebased theory. Journal of Management Studies. Vol. 33, $N^{\circ}$ 6. Pág. 757-785.

NELSON, R.R. y WINTER, S.G. (1982): An Evolutionary Theon' of Economic Change. Massachusets: The Belknap Press of Harvard University Press. Pág. 14.

PENROSE, E. (1959): The theory of the grouth of the firm. Oxford: University Press.

PETERAF, M.A. (1993): "The cornerstones of competitive advantage: A resource-based viewn, Strategic Management Journal. Vol. 14. Pág. 179-191.

PORTER, Michael (1985): Ventaja Competitiva. México: Cecsa.

POWELL, T. C. y DENT-MICALLEF, A. (1997): "Information Technology as Competitive Advantage: The Role of Human, Business, and Technology Resources. Strategic Management Journal. Vol. 18, No 5. Pág. 375-405.

PRAHALAD, C.K. y HAMEL, G. (1990): "The core competence of the corporation" Harvard Business Review. No 68. Pág. 79-91.

PRAHALAD, C.K. y HAMEL, G. (1991): "La organización por unidades estratégicas de negocio ya no sirve" Harvard Deusto Business Review: $1^{\circ}$ trimestre. Pág. 47-64.

REED, R. y DeFILIPPI, R. (1990): "Causal ambiguity, barriers to imitation and sustainable competitive advantage" Academy' of Management Revieu'. Vol. 15, № 1. Pág. 88-102. RICARDO, D. (1891): Principles of Political Economy and Taxation. Londres: G. Bell. RUMELT, R.P.; SCHENDEL, D. y TEECE, D.J. (Eds.)(1994):

Fundamental Issues in Strategy. Boston: Harvard Business School Press.

RUMELT, R.P., SCHENDEL, D. y TEECE, D.J. (1991): "Strategic management and economics" Strategic Management Journal. Vol. 12. Pág. 5-29.

RUMELT, R.P. (1991): "How much does industry matter?", Strategic Management Journal. Vol. 12. Pág. 143.

SANCHEZ BARRIGA, F. (1993): Técnicas de administración de recursos bumanos. MéxiCO: Limusa.

SALAS FUMÁS, V. (1996): "Economía y gestión de los activos intangibles", Economía Industrial, $\mathrm{N}^{\circ}$ 307. Pág. 17-24.

SCHNEIDER, Benjamin y BOWEN, David E. (1993): “Los recursos humanos en la calidad del servicio" Harvard Deusto Business Revieu. No 57 . Mayo. Pág. 69 y ss. 
SCHULZE, W.S. (1994): "The Two Schools of Thought in Resource-based Theory: Definitions and Implications for Research", Advances in Strategic Management. Vol. 10A. Pág. 127-151.

SCHULER, R.S. y JACKSON, S.E (1987):"Linking competitive strategieswith human resource management practices" Academy of Management Executive. Vol 1. № 3. Pág. 207-219

SELZNICK, P. (1957): Leadership in Administration: A Sociological Interpretation. Nueva York: Harper \& Row.

SILEM, Ahmed y MARTINEZ, Gérard (1983): Information des salariés et stratégies de. communication. París: Les Editions d'Organisation.

SWIERCZ, Paul Michael y SPENCER, Barbara A. (1992): "HRM and sustainable competitive advantage: lessons from Delta Air Lines"

Human Resource Planning. Vol. 15, No 2. Pág. 36-43.

TEECE, David J.; PISANO, Gary y SCHUEN, Amy (1997): "Dynamic Capabilities and Strategic Management" Strategic Management Journal. Vol. 18, No 7. Pág. 509-533.

TEECE, David J. (1982): "Towards an Economic Theory of the Multiproduct Firm" Jour nal of Economics Behavior and Organization. Vol. 3. Pág. 39-63.

THIERRY, Dominique (1994): "La place réelle des ressources humaines dans les stratégies d'entreprise" Revue française de gestion. N97, Enero-Febrero. Pág. 40-53.

THOMPSON, A.A. y STRICKLAND, A.J. (1990): Strategic Management: Concept and Cases. Illinois: Irwin Homehood.

ULRICH, Dave (1989a): "Tie the corporate knot: Gaining complete customer commitment. Sloan Management Review. Verano. Pág. 19-28.

ULRICH, Dave (1989b): “Assessing human resource effectiveness: Stakeholder, index and relationship approaches" Human Resource Planning. Vol. 12. Pág. 301-315.

ULRICH, Dave and LAKE, D. (1990): Organizational capability: Competing from the inside/out. New York: Wiley.

VATEVILLE, E. (1985): Mesure des Ressurces Humaines et Gestion de l'Entreprise. París: Economica.

VENTURA VICTORIA, J. (1996): Análisis dinámico de la estrategia empresarial: un ensayo interdisciplinar. Servicio Publicaciones Universidad de Oviedo.

WALKER, Alfred J. (1997): "Technology: A Catalytic Converter of HR to the Wired Global World". Human Resources Information Systems Catalogue Page [WWW page]. URL: http://www.hronline.com/lib/hris.html.

WERNERFELT, B. (1984): "A Resource-based View of the Firm", Strategic Management Journal. Vol. 5. Pág. 171-180.

WRIGHT, P.M.; MCMAHAN, G.C. y McWILLIAMS, A. (1994): "Human resources and sustained competitive advantage: a resource-based perspectiven International Journal of Human Resource Management. Vol. 5 № 2. Mayo. Pág. 301-326

YEUNG, A. K. y BERMAN, B. (1997): "Adding value through human resources" Human Resource Management. Vol. 36, No 3. Pág. 321-335. 